\title{
Study of the Most Appropriate Routing Protocol based on between the Source Node and the Sink Node
}

\author{
Cheng Huang and Wei-wei Shao \\ School of Automation, Nanjing University of Science and Technology, Nanjing, \\ China \\ hearthc@163.com
}

\begin{abstract}
Random distribution of sensor nodes in the wireless sensor network easily causes information transmission congestion, transmission delay, uneven network energy consumption and shorter network lifetime. This paper gives full consideration to the residual energy, the network lifetime and the amount of data transmission, then proposes the most appropriate routing protocol when comparing the traditional minimum hops algorithm, which is based on between the source node and the sink node. The algorithm has a simple structure and is easy to implement, which can be effectively applied to the medium-scale wireless sensor networks. In addition, this algorithm adopts multiple-hop mechanism, which effectively saves more energy. The simulation results show that this algorithm has a good effect on energy saving and the amount of data transmission. Therefore, the network has more survival nodes and extends the network lifetime.
\end{abstract}

Keywords: Energy Saving; Multiple-hop;Minimum hops algorithm

\section{Introduction}

The traditional plane routing protocols [1-3] in the small and medium scale wireless network increase the network energy consumption and shorten the network lifetime, which have some serious impacts on the efficiency of sensor nodes information collection. The key of routing algorithm in high real-time applications is the rapidity and reliability of forwarding information from the source sensor node to the sink node. With the continuous in-depth study of routing algorithm, the network can effectively collect, process and forward information for the needs of people [5-6].

Nowadays, wireless sensor networks [7] are widely used in industrial production, environmental monitoring, military, agriculture, medical care and people's daily life. Which put forward higher requirements to reliability, real-time, accuracy and robustness. So the routing algorithm is an important research in the wireless sensor networks [8].

This paper proposes the most appropriate routing protocol based on between the source node and the sink node (MARP), which aims to find a suitable path for forwarding information from the source node to the sick node. This algorithm has a simple network topology and is easy to implement, which effectively saves more energy for network. Thus this algorithm can extend the network lifetime and increase the amount of data transmission.

\section{MARP Network Model and Energy Model}

\subsection{Network Model}

In order to analyze and study the performance of the algorithm conveniently, the number of nodes in the network is $\mathrm{N}$ in this paper, which randomly distribute in the $\mathrm{a}^{*} \mathrm{a}$ square area. Where each sensor node is independent of others and has the same initial 
energy. In addition, the network is homogeneous, which has the same ability to send and receive data. As showed in figure 1: all the nodes in the network are static, and the base station is located in the center of network.



Figure 1. The Topological Structure of the Network

\subsection{Energy Model}

In order to analyze and study the performance of MARP and compare the minimum hops algorithm conveniently in this paper, we use the first order radio model in terms of node energy consumption in paper [11]. As showed in figure 2.



Figure 2. The First Order of Radio Energy Model Figure

Where $\mathrm{E}_{\mathrm{Tx}}(\mathrm{k}, \mathrm{d})$ represents the amount of energy consumption for sending $\mathrm{k}$ packages, $E_{R X}(k)$ represents the amount of energy consumption for receiving $\mathrm{k}$ packages, $\boldsymbol{\varepsilon}_{\text {amp }}$ represents the power amplifier to send data, $\mathrm{d}$ is the signal transmission distance. $d^{r}$ represents the decline coefficient of energy in the processing of signal transmission, $d_{0}$ is a distance threshold, which is a constant and determined by the environment in energy attenuation model. If the transmission distance $d$ is less than the distance threshold, then the free space model, $\mathrm{r}=2$, is used, otherwise using the multiple-path attenuation model, $r=4$.

Assuming the communication channel is two-way symmetrical, when the sender sends $\mathrm{k}$-bit data to the receiver and the receiver receives $\mathrm{k}$-bit data, the energy consumption is:

$$
\begin{gathered}
\mathrm{E}_{\mathrm{Tx}}(\mathrm{k}, \mathrm{d})=\mathrm{E}_{\mathrm{Tx}-\text { else }}(\mathrm{k})+\mathrm{E}_{\mathrm{Tx}-\mathrm{amp}}(\mathrm{k}, \mathrm{d})=\left\{\begin{array}{l}
\mathrm{k}^{*} \mathrm{E}_{\text {elec }}+\mathrm{k}^{*} \varepsilon_{\mathrm{fs}} * \mathrm{~d}^{2}, \mathrm{~d}<\mathrm{d}_{0} \\
\mathrm{k}^{*} \mathrm{E}_{\text {elec }}+\mathrm{k} * \varepsilon_{\mathrm{mp}} * \mathrm{~d}^{4}, \mathrm{~d} \geq \mathrm{d}_{0}
\end{array}\right. \\
E_{R x}(k)=E_{R x-\text { else }}(k)=k * E_{\text {elec }}
\end{gathered}
$$

Where $\mathrm{E}_{\mathrm{TX}-\mathrm{el} \mathrm{se}}$ represents the energy consumed by the internal circuit of sensor node during transmitting unit data, while $E_{R x-e 1 s e}$ represents the energy consumed by the internal circuit of sensor node during receiving unit data. $\varepsilon_{\mathrm{fs}}, \varepsilon_{\mathrm{mp}}$ represents the signal 
amplification parameter of the free space model and the signal amplification parameter of the multiple-path attenuation model respectively, $\mathrm{E}_{\mathrm{Tx}-\mathrm{amp}}$ represents the energy consumed by sender through the amplifier.

\section{The Relative Description of Minimum Hops Algorithm and MARP}

\subsection{Minimum Hops Algorithm}

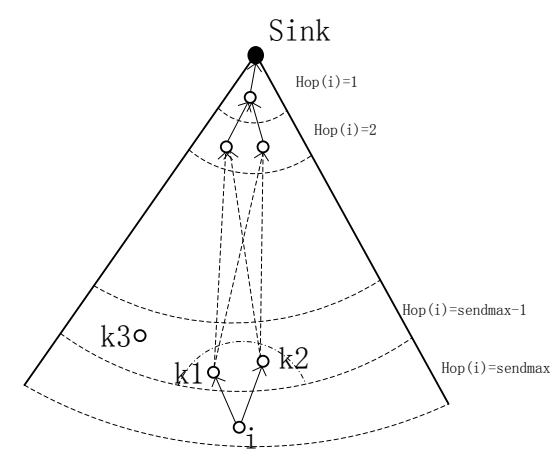

\section{Figure 3. Minimum Hops Algorithm Routing Path}

Where the minimum hop distance is 0.75 times of the sensor node communication radius, which aims to prevent more isolated nodes, thus improving the possibility of finding the next node in the next hop. According to this way to continue until reaching the sink node. The algorithm has added the communication radius of network node and the minimum hop distance, but the simulation results show that the network energy consumption is relatively large and the network lifetime is relatively short.

The minimum hops algorithm includes two stages: establishing the gradient field and transmitting packets.

(1) The gradient field is established through the way of flooding to transmit control packets. The control packets are consisted of node ID, residual energy and the value of hop. Initializing the hop of each node for 1000 and the sink node for 0 before establishing the gradient field, then flooding is started. If the node receives the hop of the control packets which is smaller than itself node, then the hop of the control packets will add 1 as the new hop of the node. Otherwise the sensor node does not accept the control packets and the hop of node is still the same. A gradient field is established after a period of time.

(2) After establishing the gradient field, the information is transmitted from the source node to the sink node through flooding. The node of collecting information is called the source node. After collecting information, the node transmits packets to its next hop node in the range of communication radius. If the hop of one node is $n$, the hop of its next hop node is $n-1$. The node receiving information continues to transmit its packets to its next hop node, until the sink node. 


\subsection{MARP}

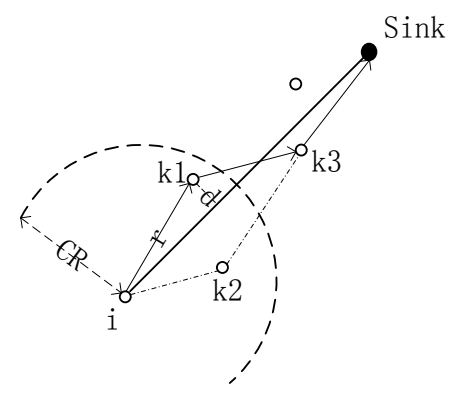

Figure. 4 MARP Routing Path

Where $\mathrm{i}$ is the source node, $\mathrm{k} 1$ is the next hop node, $\mathrm{r}$ is the distance between $\mathrm{k} 1$ and $\mathrm{i}$, $\mathrm{d}$ is the distance from the node of $\mathrm{k} 1$ to the line which through $\mathrm{i}$ and sink.

As shown in the Figure 4. The node is selected as the next hop node, which has more remaining energy and a longer distance to the source node $i$ and a shorter distance to the sink node in the range of source node communication radius CR. So the next hop node of $\mathrm{i}$ is the node $\mathrm{k} 1$ instead of the node $\mathrm{k} 2$, and the selecting routing path is as the thin line.

This algorithm adds the remaining energy, $r$ and $d$ in choosing the next hop node. So the reference value is obtained just as the function (3):

$$
\text { reference }=\alpha * \frac{E(k)}{E_{o}}+\beta * \frac{r(k)}{C R}+\lambda *\left(1-\frac{d}{C R}\right)
$$

The value of weight coefficient $\alpha, \beta, \lambda$ are $0.1,0.45,0.45$ respectively.

\section{Protocol Simulation Result and Analysis}

\subsection{The Simulation Parameters Setting}

The simulation conditions of MARP and minimum hops algorithm in this paper are shown in table 1 . Network space: square of $200 \times 200$, the sensor nodes initial energy $0.5 \mathrm{~J}$.

Table 1. The Simulation Parameters Setting

\begin{tabular}{|c|c|c|c|}
\hline Parameters & Values & Parameters & Values \\
\hline $\begin{array}{c}\text { The number of node }: \mathrm{N} \\
\begin{array}{c}\text { The sink node } \\
\text { coordinate } \\
\text { distance }\end{array}\end{array}$ & $\begin{array}{c}(0 \mathrm{~m},, 0 \mathrm{~m}) \\
\boldsymbol{\varepsilon}_{m p}\end{array}$ & $0.0013 \mathrm{pJ} / \mathrm{bit} / \mathrm{m}^{4}$ \\
\hline Packet length & $4000 \mathrm{bit}$ & $\boldsymbol{\varepsilon}_{f s}$ & $10 \mathrm{pJ} / \mathrm{bit} / \mathrm{m}^{2}$ \\
\hline Control packet length & $100 \mathrm{bit}$ & $E_{\text {elec }}$ & $50 \mathrm{pJ} / \mathrm{bit}$ \\
\hline Communication radius & $25 \sqrt{2}$ & & \\
\hline
\end{tabular}

We use the MATLAB software in the simulation experiment of MARP and minimum hops algorithm in this paper. In order to compare and analyze the performance of the protocols, the experimental simulation is carried out from the following four aspects: the number of network remaining nodes, the network overall energy consumption, the network lifetime and the amount of packets received by the sink node. If all the neighbor 
nodes of one node are dead in the network, then the node will become the isolated node. The isolated node will be also regarded as the dead node. When the number of dead nodes in network reaches half of the total number of network nodes, this network will be failure. The performance of network is evaluated by the first node dead (FND) and the half node dead (HND).

\subsection{The Analysis of Simulation Results}

(1) The number of remaining nodes in network changes over time. As shown in Figure 5 , the remaining nodes of MARP and minimum hops algorithm change over time.

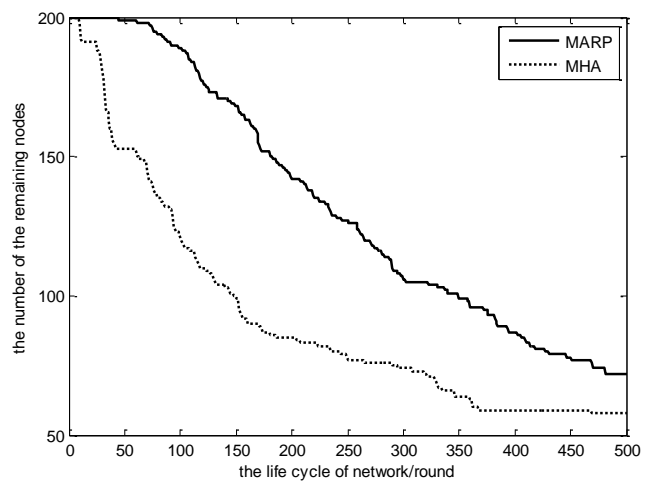

Figure 5. The Number of Remaining Nodes Changes Over Time

According to the simulation results of the Figure 5, the FND of minimum hops algorithm is 9, the HNF of minimum hops algorithm is 147; while the FND of MARP is 45, the HND of MARP is 350. We can see the intuitive comparison of minimum hops algorithm and MARP by the Figure 6 .



Figure 6. The Comparison of Protocols Life Cycle

As shown in Figure 6, the comparison of network life cycle between minimum hops algorithm and MARP. We can know that the lifetime of MARP is longer than the minimum hops algorithm one obviously. When comparing to minimum hops algorithm, MARP's FND and HND are extended by 5 times, 2.38 times respectively. The experimental simulation results show that MARP extends the network lifetime effectively.

(2) The network overall energy consumption changes over time. As shown in Figure 7: the network overall energy consumption of minimum hops algorithm and MARP changes over time. 


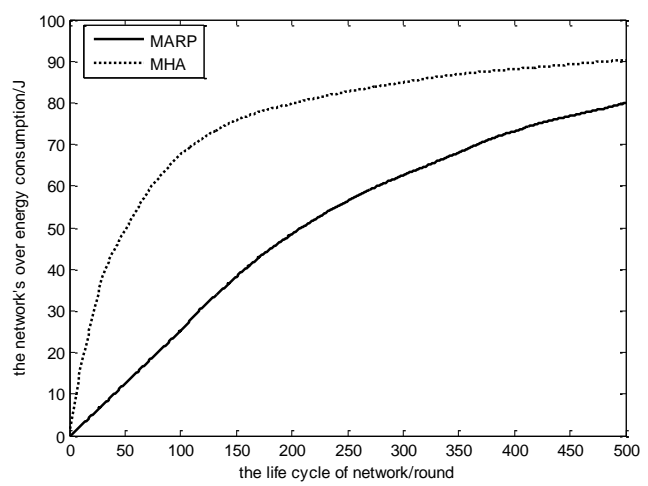

Figure 7. The Network's Overall Energy Consumption Changes over Time

According to the simulation results of the network overall energy consumption in the Figure 7, MARP has much lower energy consumption and saves more energy for the whole network than minimum hops algorithm in the same situation. The intuitive comparison for energy consumption is shown in the Figure 8.

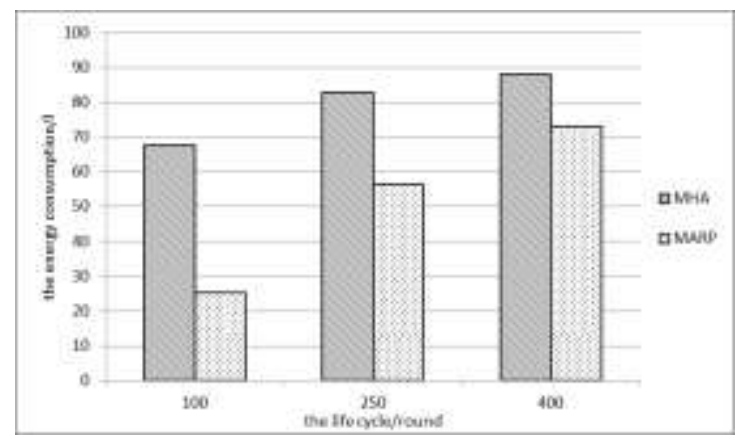

Figure 8. The Energy Consumption of different Rounds

The Figure 8 shows that the comparison of the network overall energy consumption between MARP and minimum hops algorithm in different rounds. The MARP consumes less energy than minimum hops algorithm. MARP's energy consumption is reduced by $62.6 \%, 31.7 \%, 16.9 \%$ respectively in the 100th round,250th round,400th round when comparing minimum hops algorithm. According to the simulation result, MARP is better than minimum hops algorithm in the aspect of network overall energy consumption.

(3) The amount of packets received by the sink node in three different stages is shown in Figure 9.

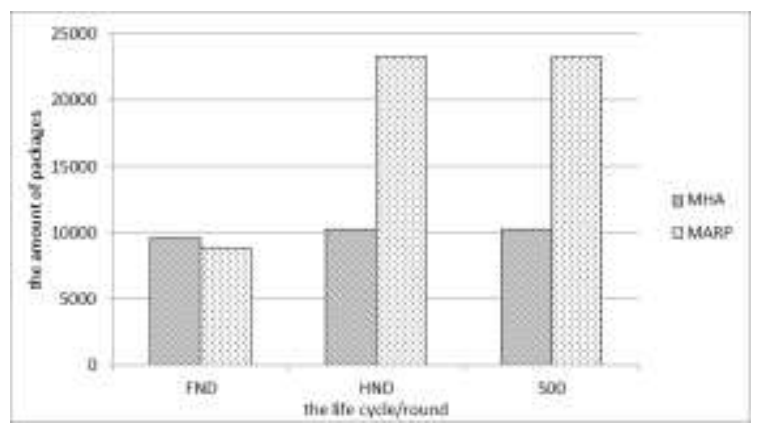

Figure 9. The Amount of Packages received by the Base Station

According to the simulation results, the amount of packets received by the base station 
is $8799,23314,23314$ respectively in the FDN, HND, 500th round, when the MARP is carried out; while minimum hops algorithm is conducting, the amount of packets received by the base station is 9575, 10245, 10245 respectively in the FDN, HND, 500th round. The intuitive comparison is shown in the fig .9, the amount of packets is close for the two kinds of protocols when the first node is dead; But the MARP's packets is 2.27 times that of the later ones when half of all nodes are dead; In addition, the amount of packets is the same for the two kinds of protocols in the 500th round. Thus after the HND round, with the increasing number of the dead nodes in the network, the routing path is interrupted so that the network is failed.

\section{Conclusion}

In this paper, the core of the MARP aims to find the most appropriate routing path between the source node and the sink node. This routing protocol has a simple network structure and is easy to implement. In addition, this algorithm gives a full consideration on the network remaining energy. So this algorithm can effectively find the most suitable node as its next hop node in the range of communication radius. As can be seen from the simulation analysis of network performance, the MARP has a better performance than minimum hops algorithm in aspect of the network lifetime, the network overall energy consumption and the transmission packets.

\section{Acknowledgments}

This work was supported by the Natural Science Foundation of Jiangsu Province (no. BK2012803), Doctoral Program of the Ministry of Education of China (no.20113219110028), and the Advantage Discipline Construction Project for University of Jiangsu Province.

\section{References}

[1] Z. Zhe, "An improved routing algorithm based on the minimum hop routing protocol for wireless sensor networks", Nanjing university of science and technology, (2008).

[2] Y. Xunhao and H. Jiaming, "The MHC-MAC protocol based on the minimum hop routing protocol", Mobile Communication, no. 14, (2013), pp. 51-56.

[3] Z. Qiang and L. Yaping, "A girding-based clustering routing algorithm for sensor networks", Zhongnan University of Forestry Science and Technology, vol. 30, no. 4, (2010), pp. 166-169.

[4] Z. Run, "Study of Clustered Energy Saving Routing Protocol for Mobile Nodes in Three-dimensional Space", Nanjing university of science and technology, (2014).

[5] C. Zhibo and X. Xiaocheng, "Improved minimum hop count routing protocol in wireless sensor network", Computer Science, vol. 40, no. 4, (2013), pp. 83-85.

[6] W. Kunchi, Z. Yuejie and X. Chen, "An improved minimum hop routing protocol for wireless sensor networks", Transducer and Microsystem Technologies, no. 8, (2012), pp. 52-56.

[7] S. Haifei, Y. Zhong and W. Jun, "Review on research status and application of wireless sensor networks", Journal of Mechanical and Electrical Engineering, no. 1, (2011).

[8] S. Limin and L. Jianzhong, "Wireless sensor networks", Beijing: Tsinghua University Press, (2005).

[9] Y. Yukun, W. Guan and R. Zhi, "Energy Balanced Clustering Algorithm for Self-Energized Wireless Sensor Networks", Chinese Journal of Sensor and Actuators, no. 10, (2013), pp. 1420-1425.

[10] T. Yu and S. Xiaoou, "An Energy-saving Geographic Routing in Wireless Sensor Network", Science Technology and Engineering, vol. 14, no. 10, (2014), pp. 61-64.

[11] W. R. Heinzelman, A. Chandrakasan and H. Balakrishnan, "An Appli- cation Specific Protocol Architecture for Wireless Microsensor Networks", IEEE Transactions on Wireless Communications, vol. 1, no. 4, (2002), pp. 660-670. 
International Journal of Control and Automation

Vol. 10, No. 6 (2017) 